\title{
PERFIL DE MORBIMORTALIDADE DE RECÉM-NASCIDOS DE RISCO
}

Christine Baccarat de Godoy Martins ${ }^{1}$, Ariane Aguillar Barcelon², Fernanda Cristina Aguiar Lima², Maria Aparecida Munhoz Gaíva ${ }^{4}$

RESUMO: Estudo descritivo analisou o perfil de morbimortalidade, nos seis primeiros meses de vida, entre neonatos de risco nascidos em Cuiabá - Mato Grosso, em janeiro de 2011. As fontes de dados foram Declaração de Nascido Vivo e inquérito domiciliar após seis meses do nascimento. O estudo foi aprovado por Comitê de Ética em Pesquisa e as famílias assinaram o Termo de Consentimento Livre e Esclarecido. Foram estudados 113 recém-nascidos, 70,8\% apresentaram morbidade no primeiro semestre de vida, principalmente doenças respiratórias e diarreicas; a morbidade foi maior entre recém-nascidos cujas mães não trabalhavam fora, adolescentes e que amamentaram por pouco tempo; $5,3 \%$ das crianças faleceram, sendo $83,3 \%$ no período neonatal; os óbitos ocorreram mais entre recém-nascidos com baixo peso ao nascer, prematuros, cujas mães não trabalhavam fora. Espera-se que os resultados subsidiem políticas específicas de atenção ao grupo de recém-nascidos vivos de risco. DESCRITORES: Recém-nascido, Grupos de risco, Morbidade infantil, Mortalidade infantil.

\section{PERFIL DE MORBIMORTALIDAD DE RECIÉN NACIDOS DE RIESGO}

RESUMEN: Estudio descriptivo que ha analizado el perfil de morbimortalidad, en los seis primeros meses de vida, entre neonatos de riesgo nacidos en Cuiabá - Mato Grosso, en enero de 2011. Las fuentes de datos fueron Declaración de Nacido Vivo e investigación domiciliar después de seis meses del nacimiento. El estudio fue aprobado por Comité de Ética en Investigación y las familias firmaron el Término de Consentimiento Libre y Esclarecido. Fueron estudiados 113 recién nacidos, 70,8\% presentaron morbidad en el primero semestre de vida, principalmente enfermedades respiratorias y diarreicas; la morbidad fue mayor entre recién nacidos cuyas madres no trabajaban fuera, adolescentes y que amamantaban por poco tiempo; $5,3 \%$ de los niños fallecieron, siendo $83,3 \%$ en el periodo neonatal; los óbitos ocurrieron más entre recién nacidos con poco peso al nacer, prematuros, cuyas madres no trabajaban fuera. Se espera que los resultados sean subsidiadas políticas específicas de atención al grupo de recién nacidos vivos de riesgo. DESCRIPTORES: Recién nacido, Grupos de riesgo, Morbidad infantil, Mortalidad infantil

\section{PROFILE OF MORBIDITY AND MORTALITY IN AT-RISK NEWBORNS}

ABSTRACT: This descriptive study analyzed the profile of morbidity and mortality in the first six months of life among at-risk neonates in Cuiabá in the state of Mato Grosso, in January 2011. The data sources were the Certificate of Live Birth and home interviews held six months after the birth. The study was approved by the Research Ethics Committee and the families signed the Terms of Free and Informed Consent. 113 newborns were studied, of whom $70.8 \%$ presented morbidity in the first six months of life, mainly involving respiratory and diarrheal diseases; morbidity was greater among newborns whose mothers did not work outside the home, who were adolescents, or who breast-fed for little time; $5.3 \%$ of the children died, of whom $83.3 \%$ died in the neonatal period; the deaths occurred more among newborns with low birth weights, or who were premature, or whose mothers did not work outside the home. It is hoped that the results may support specific policies for care to the group composed of live at-risk newborns.

DESCRIPTORS: Newborn; Risk groups; Child morbidity; Child mortality.

\footnotetext{
${ }^{1}$ Enfermeira. Pós-Doutora em Saúde Pública. Professora da Universidade Federal de Mato Grosso. Cuiabá-MT-Brasil

${ }^{2}$ Acadêmica de Enfermagem. Universidade Federal de Mato Grosso. Cuiabá-MT-Brasil

${ }^{3}$ Enfermeira. Mestranda em Enfermagem.Universidade Federal de Mato Grosso. Cuiabá-MT-Brasil

${ }^{4}$ Enfermeira. Pós-Doutora em Enfermagem em Saúde Pública. Professora da Universidade Federal de Mato Grosso. Cuiabá-MT-Brasil
}

Autor correspondente:

Recebido: $25 / 05 / 2013$

Christine Baccarat de Godoy Martins

Aprovado: 03/02/2014

Universidade Federal de Mato Grosso

Rua Fortaleza, 70 - 78065-350 - Cuiabá-MT-Brasil

E-mail: leocris2001@terra.com.br 


\section{INTRODUÇÃO}

Os indicadores de morbimortalidade de crianças são fundamentais para avaliar a qualidade de atenção à saúde materno-infantil, bem como para a promoção da saúde da população e prevenção de doenças ${ }^{(1-2)}$. Neste sentido, o Brasil vem progredindo na redução da mortalidade infantil, mais expressivamente pela redução da mortalidade pós-neonatal (27 a 364 dias de vida $)^{(3-4)}$. Atribui-se este fato a ações de baixo custo e alta eficácia como, por exemplo, o uso da Terapia de Reidratação Oral (em especial, em casos de diarreia), controle das infecções respiratórias agudas, incentivo ao aleitamento materno, melhoria no saneamento básico, aumento da cobertura vacinal, expansão dos serviços básicos de saúde, entre outras ${ }^{(5)}$.

Ainda assim, os índices de mortalidade infantil são elevados no país, principalmente nos primeiros dias de vida ( 0 a 27 dias de vida), o que faz com que os neonatos mereçam atenção especial, principalmente os nascidos em condições consideradas de risco ${ }^{(4,6)}$.

O Ministério da Saúde classifica como recém-nascido de risco o que responde a, pelo menos, um dos seguintes critérios: baixo peso ao nascer (menos de $2.500 \mathrm{~g}$ ); idade gestacional inferior a 37 semanas; asfixia grave (Apgar menor que sete no $5^{\circ}$ minuto de vida); recém-nascido de mãe adolescente (menos de 18 anos); hospitalização ou intercorrência na maternidade ou unidade de assistência ao recém-nascido; necessidades de orientações especiais à alta da maternidade; recém-nascido de mãe com menos de 8 anos de escolaridade; residir em área de risco e história de morte de criança menor de 5 anos na família ${ }^{(4)}$.

Além da mortalidade, é de fundamental importância analisar também os determinantes de morbidade neste grupo, uma vez que o reconhecimento precoce e a prevenção de doenças contribuem para redução dos óbitos. Nesse sentido, o perfil epidemiológico aponta que as doenças respiratórias são o primeiro motivo de consulta em ambulatórios e serviços de urgência infantis, sendo a pneumonia e a diarreia as principais doenças em crianças menores de um ano ${ }^{(4)}$. Vale destacar que recém-nascidos prematuros são mais vulneráveis a desenvolver morbidades que os recém-nascidos a termo ${ }^{(7-8)}$. Autores ainda apontam a condição socioeconômica, a exemplo baixa renda familiar e escolaridade materna, como fator diretamente relacionado às condições de saúde infantil ${ }^{(9)}$.

Reconhecendo a importância desses indicadores, principalmente entre os recém-nascidos de risco, o presente estudo objetivou analisar o perfil de morbimortalidade, nos seis primeiros meses de vida, dos recém-nascidos de risco nascidos no Município de Cuiabá - Mato Grosso, Brasil.

\section{MÉTODO}

Estudo descritivo, quantitativo, cuja população foi composta por neonatos de risco nascidos em Cuiabá - Mato Grosso, em janeiro de 2011. A primeira fonte de dados consistiu na Declaração de Nascido Vivo, utilizada para identificar e incluir os recém-nascidos na pesquisa, de acordo com os critérios de classificação definidos pelo Ministério da Saúde para recém-nascido de risco. Do total de nascimentos em janeiro de 2011 (1.107), foram selecionados 113 recém-nascidos de risco que obedeciam aos critérios de inclusão.

Após seis meses do nascimento da criança foi realizado inquérito domiciliar, utilizando-se instrumento fechado. Neste momento, as mães foram convidadas a participar da pesquisa, assinando o Termo de Consentimento Livre e Esclarecido.

No inquérito domiciliar foram coletadas as seguintes variáveis: peso ao nascer e sexo do recém-nascido; história obstétrica materna (número de consultas pré-natal, duração da gestação do recém-nascido em estudo); histórico de morbidade (ocorrência de doenças até os seis meses de idade, quais doenças, se foi necessário internação por doença e tempo de internação) e de mortalidade do recém-nascido (ocorrência de óbito nos seis primeiros meses de idade, se a família procurou por serviço de saúde até 24 horas antes do óbito, se o recém-nascido foi internado antes do óbito, o tempo de internação antes do óbito, o local, a idade e a causa do óbito, baseada no código da CID-10 constante na Declaração de Óbito); com quem o recém-nascido residia; variáveis da mãe (situação ocupacional, escolaridade, idade); acompanhamento do crescimento e desenvolvimento pela Unidade de Saúde (se realizava ou não); situação vacinal da criança (se estava em dia ou em atraso); frequência com que a mãe procura o serviço de saúde (esporádica, mensal, quinzenal, semanal, não procura, ignorado); duração do aleitamento materno exclusivo para o recém-nascido em estudo.

A análise de dados se deu pelo programa EpiInfo, versão 3.5.2., aplicou-se o teste do qui-quadrado, considerando o valor de $p<0,05$ para associação estatisticamente significativa.

O estudo faz parte da pesquisa "Avaliação da atenção à criança na Rede Básica de Saúde de Cuiabá 
- Mato Grosso, com ênfase em sua organização e assistência, e nas práticas de enfermagem", aprovada pelo Comitê de Ética em Pesquisa do Hospital Universitário Júlio Muller-Universidade Federal de Mato Grosso, sob o protocolo 882/CEP-HUJM/2010.

\section{RESULTADOS}

Dos 113 recém-nascidos de risco pesquisados, $70,8 \%$ apresentaram doença até os seis meses de idade, destacando-se as respiratórias com 43,3\% (gripe, inflamação da garganta, bronquite, bronquiolite, infecção de trato respiratório e resfriado) e doenças diarréicas com 29,2\% (Tabela 1). Entre os que apresentaram morbidade, 12,5\% (10) necessitaram de internação. Entre estes, $60,0 \%(6)$ necessitaram permanecer de 1 a 3 dias no hospital, $30 \%$ (3) de 10 dias e mais, e $10 \%$ (1) de 4 a 6 dias de hospitalização.

Buscaram-se associações entre morbidade até os seis meses de idade com a situação ocupacional, a escolaridade e a idade da mãe, com quem a criança residia, acompanhamento de crescimento e desenvolvimento, imunização, frequência com que a mãe buscava por atendimento na UBS e aleitamento materno. O maior percentual de morbidade se deu entre as crianças cujas mães não estavam inseridas no mercado de trabalho (73,8\%), seguido por aquelas cujas mães estavam inseridas no mercado informal $(16,3 \%)$ e no mercado formal $(7,5 \%)(p=0,4987)$.

As crianças que moravam com o pai e a mãe foram as que apresentaram maior percentual de doenças até seis meses de idade $(56,3 \%)$, seguidas pelas que residiam com a mãe $(28,8 \%)$, outras pessoas $(11,3 \%)$, parente $(2,5 \%)$, mãe e padrasto $(1,3 \%)(\mathrm{p}=0,0000)$.

A morbidade entre as crianças aumentou na medida em que aumenta a escolaridade da mãe, com maior percentual de crianças doentes entre mães com 8 a 11 anos de estudo (52,5\%), seguidas por aquelas com 4 a 7 anos de escolaridade (43,8\%), 1 a 3 anos $(2,5 \%)$ e tempo de estudo ignorado $(1,3 \%)(\mathrm{p}=0,9381)$. Houve maior percentual de morbidade entre as crianças cujas mães tinham menos de 18 anos de idade (41,3\%), seguidas pelas mães com 18 a 27 anos (31,3\%), 28 a 37 $\operatorname{anos}(25,0 \%)$ e 38 a 47 anos $(2,5 \%)(p=0,3159)$.

Tanto as crianças que fazem acompanhamento na UBS como em outra instituição apresentaram alta porcentagem de morbidade $(74,7 \%$ e $71,4 \%$, respectivamente $)(\mathrm{p}=0,0000)$. A maioria das crianças, tanto as que adoeceram como as que não adoeceram, estavam com a imunização em dia $(63,8 \%$ e $64,3 \%$, respectivamente $)(p=0,0000)$. A maior proporção de crianças que adoeceram se deu entre aquelas cujas mães procuravam por atendimento no serviço de saúde esporadicamente $(38,8 \%)$, seguidas pelas de procura mensal $(32,5 \%)$, a não procura $(21,3 \%)$, procura quinzenal $(2,5 \%)$, procura semanal $(1,3 \%)$ e frequência ignorada $(3,6 \%)(\mathrm{p}=0,0000)$.

O percentual de morbidade se mostrou reduzido conforme aumentavam os meses de aleitamento materno exclusivo (Tabela 2). Entre as que receberam aleitamento materno exclusivo até seis meses de idade, a morbidade reduziu significativamente para $2,5 \%(\mathrm{p}=0,0000)$.

Da população de estudo, 5,3\%(6) foram a óbito, $83,3 \%$ (5) no período neonatal e $16,7 \%$ (1) no período pós-neonatal. Destes, $66,7 \%$ (4) eram do sexo masculino $(\mathrm{p}=0,5339) .33,3 \%(2)$ dos responsáveis não procuraram o serviço de saúde até 24 horas antes do óbito e 66,6\%(4) dos recém-nascidos nem chegaram a ter alta hospitalar após o nascimento, com o óbito ocorrendo ainda no hospital. Quanto às causas do óbito, $50,0 \%$ (3) das mães não souberam relatar e não estavam com a Declaração de Óbito em mãos no momento do inquérito domiciliar; $16,7 \%(1)$ se deram por má formação cerebral; $16,7 \%(1)$ por septicemia e Síndrome de Down; e 16,7\%(1) por septicemia, insuficiência respiratória aguda e prematuridade.

Buscou-se associação entre óbito do $\mathrm{RN}$ até os seis meses de idade com: situação ocupacional, escolaridade e idade da mãe, número de consultas pré-natal, idade gestacional, peso ao nascer e sexo do RN. O maior percentual de óbitos ocorreu entre as crianças cujas mães não estavam inseridas no mercado de trabalho $(50 \%)$, seguidas pelas que trabalham com vínculo informal $(33,3 \%)$ e formal $(16,7 \%)(p=0,5553)$. Entre as crianças que vieram a óbito, $50 \%$ das mães tinham entre 4 e 7 anos de estudo e $50 \%$ de 8 a 11 anos de escolaridade $(\mathrm{p}=0,9731)$.

Entre os recém-nascidos que faleceram, $50 \%$ eram filhos de mães com idade entre 28 e 37 anos; $33,3 \%$ de mães entre 18 a 27 anos; e 16,7\% de mães menores de 18 anos $(\mathrm{p}=0,5128)$. O maior percentual dos óbitos se deu entre as mães que realizaram 6 ou mais consultas pré-natais (50\%), seguidas pelas que tiveram menos de 6 consultas (33,3\%), sendo que em $16,7 \%$ delas não foi possível conhecer o número de consultas pré-natal $(\mathrm{p}=$ $0,3648)$. A prematuridade obteve o maior percentual de óbito $(66,7 \%)(\mathrm{p}=0,0603)$ (Tabela 3).

Quanto menor o peso ao nascer, maior o percentual de óbito entre $66,7 \%$ que nasceram pesando menos que $2.500 \mathrm{~g}$ e $33,3 \%$ pesando entre $2.500 \mathrm{~g}$ a $3.500 \mathrm{~g}$ (Tabela 4) $(\mathrm{p}=0,0142)$. 
Tabela 1 - Distribuição dos neonatos de risco, nascidos em janeiro de 2011, segundo a morbidade até os seis meses de idade. Cuiabá-MT-Brasil, 2011

\begin{tabular}{lcccccccc}
\hline \multirow{2}{*}{ Doenças até seis meses de idade } & \multicolumn{2}{c}{ Sim } & \multicolumn{2}{c}{ Não } & \multicolumn{2}{c}{ Nãosoube referir } & \multicolumn{2}{c}{ Total } \\
& n & \% & n & \% & n & \% & n & \% \\
\hline Doenças respiratórias & 49 & 43,3 & 59 & 52,2 & 5 & 4,4 & 113 & 100 \\
Doença diarreica & 33 & 29,2 & 75 & 66,3 & 5 & 4,4 & 113 & 100 \\
Conjuntivite & 13 & 11,5 & 95 & 84,0 & 5 & 4,4 & 113 & 100 \\
Pneumonia & 12 & 10,6 & 96 & 84,9 & 5 & 4,4 & 113 & 100 \\
Anemia & 11 & 9,7 & 97 & 85,8 & 5 & 4,4 & 113 & 100 \\
Icterícia & 11 & 9,7 & 97 & 85,8 & 5 & 4,4 & 113 & 100 \\
Doenças infectocontagiosas & 7 & 6,1 & 101 & 89,3 & 5 & 4,4 & 113 & 100 \\
Alergia & 3 & 2,6 & 110 & 97,3 & - & - & 113 & 100 \\
Desnutrição & 2 & 1,7 & 106 & 93,8 & 5 & 4,4 & 113 & 100 \\
Desidratação & 2 & 1,7 & 106 & 93,8 & 5 & 4,4 & 113 & 100 \\
Otite & 2 & 1,7 & 111 & 98,2 & - & - & 113 & 100 \\
Sarampo & 1 & 0,8 & 112 & 99,1 & - & - & 113 & 100 \\
Catapora & 1 & 0,8 & 112 & 99,1 & - & - & 113 & 100 \\
Refluxo gástrico & 1 & 0,8 & 102 & 90,2 & - & - & 113 & 100 \\
Outras & 1 & 0,8 & 102 & 90,2 & - & - & 113 & 100 \\
\hline
\end{tabular}

Tabela 2 - Distribuição dos neonatos de risco, nascidos em janeiro de 2011, segundo a prevalência do aleitamento materno e a incidência de doenças até os seis meses de idade. Cuiabá-MT-Brasil, 2011

\begin{tabular}{|c|c|c|c|c|}
\hline \multirow{2}{*}{$\begin{array}{l}\text { Idade em que prevaleceu o aleitamento } \\
\text { materno exclusivo }\end{array}$} & \multicolumn{4}{|c|}{$\begin{array}{c}\text { Ocorrência de doenças nos seis primeiros meses de vida } \\
\text { Sim }\end{array}$} \\
\hline & $\mathbf{n}$ & $\%$ & $\mathbf{n}$ & $\%$ \\
\hline Não mamou exclusivamente ao seio & 20 & 25,0 & 4 & 14,3 \\
\hline Menos de 15 dias & 16 & 20,0 & 2 & 7,1 \\
\hline 15 a 29 dias & 2 & 2,5 & - & - \\
\hline 1 mês & 13 & 16,3 & - & - \\
\hline 2 meses & 8 & 10,0 & 4 & 14,3 \\
\hline 3 meses & 8 & 10,0 & 7 & 25,0 \\
\hline 4 meses & 7 & 8,8 & 5 & 17,9 \\
\hline 5 meses & 3 & 3,8 & 2 & 7,1 \\
\hline 6 meses & 2 & 2,5 & 4 & 14,3 \\
\hline Ignorado & 1 & 1,3 & 5 & - \\
\hline Total & 80 & 100 & 33 & 100 \\
\hline
\end{tabular}

Tabela 3 - Distribuição dos neonatos de risco, nascidos em janeiro de 2011, segundo a idade gestacional e a mortalidade até os seis meses de idade. Cuiabá-MT-Brasil, 2011

\begin{tabular}{lcccc}
\hline \multirow{2}{*}{ Idade gestacional } & \multicolumn{2}{c}{ Sim } & \multicolumn{2}{c}{ Óbito } \\
& $\mathbf{n}$ & $\mathbf{\%}$ & $\mathbf{n}$ & $\mathbf{\%}$ \\
\hline$<37$ semanas & 4 & 66,7 & 28 & 26,2 \\
37 a 42 semanas & - & - & 59 & 55,1 \\
42 e mais semanas & - & - & 1 & 0,9 \\
Ignorado & 2 & 33,3 & 19 & 17,8 \\
\hline Total & $\mathbf{6}$ & $\mathbf{1 0 0}$ & $\mathbf{1 0 7}$ & $\mathbf{1 0 0}$ \\
\hline
\end{tabular}


Tabela 4 - Distribuição dos neonatos de risco, nascidos em janeiro de 2011, segundo o peso ao nascer e a mortalidade até os seis meses de idade. Cuiabá-MT-Brasil, 2011

\begin{tabular}{lcccc}
\hline \multirow{2}{*}{ Peso ao nascer } & \multicolumn{2}{c}{ Óbito } & Não \\
& n & $\mathbf{\%}$ & $\mathbf{n}$ & $\mathbf{\%}$ \\
\hline$<2.500 \mathrm{~g}$ & 4 & 66,7 & 16 & 15,0 \\
$2.500 \mathrm{a} 3.500 \mathrm{~g}$ & 2 & 33,3 & 77 & 72,0 \\
$>3.500 \mathrm{~g}$ & - & - & 13 & 12,1 \\
Ignorado & - & - & 1 & 0,9 \\
\hline Total & $\mathbf{6}$ & $\mathbf{1 0 0}$ & $\mathbf{1 0 7}$ & $\mathbf{1 0 0}$ \\
\hline
\end{tabular}

\section{DISCUSSÃO}

Estudos apontam a grande ocorrência de doenças respiratórias em crianças até um ano de idade ${ }^{(10)}$, chegando a representar $36,23 \%$ das internações hospitalares, pelo Sistema Único de Saúde no Brasil ${ }^{(11)}$. A ocorrência de doença diarreica também coincide com pesquisa em que esse agravo foi a segunda causa de morte infantil nas idades de 4 a 9 meses ${ }^{(12)}$. Considerando que as doenças respiratórias e diarreicas persistem como graves problemas para a saúde infantil, tornam-se essenciais ações intersetoriais que visem melhorias gerais de vida, com ênfase no emprego e renda, no saneamento básico ${ }^{(4,9)}$. E limpeza urbana, bem como a pavimentação das ruas e cuidados com o meio ambiente, a fim de minimizar a ocorrência de doenças respiratórias nos períodos em que a umidade do ar se encontra baixa.

Vale destacar a importância das unidades básicas de saúde em realizar o diagnóstico e tratamento adequados, a fim de evitar a internação e o óbito. Este é o objetivo das atuais políticas de saúde voltadas à criança, como a estratégia Atenção Integral às Doenças Prevalentes da Infância ${ }^{(13)}$. Criada a fim de modificar o perfil de morbimortalidade infantil no país, esta estratégia caracteriza-se por condutas preconizadas relativas à promoção, prevenção e tratamento dos problemas infantis mais frequentes, como os relacionados ao aleitamento materno, à alimentação saudável, ao crescimento e desenvolvimento, à imunização, mantendo assim sob controle os agravos mais comuns na infância como a desnutrição, as doenças diarreicas, infecções respiratórias agudas e malária ${ }^{(13)}$.

Chama a atenção o fato de, entre as crianças que ficaram doentes, a maioria das mães não estarem inseridas no mercado de trabalho e ter menos que 18 anos de idade, dados que suscitam duas reflexões: a primeira é que a simples presença da mãe em casa, por não trabalhar fora do lar, não evita a ocorrência de doenças para a criança. Neste sentido, destacam-se como primordiais a melhoria nas condições socioeconômicas e gerais de vida, como a educação e acesso a serviços de saúde resolutivos. A segunda reflexão é que há necessidade de acompanhamento de saúde mais específico para o binômio mãe-filho quando se tratar de mãe adolescente, uma vez que a falta de experiência e a imaturidade constituem fatores determinantes para o cuidado da criança ${ }^{(9,14)}$.

Apesar das crianças terem acompanhamento do crescimento e desenvolvimento nas unidades de saúde, isto, por si só, não evitou os episódios de doenças, o que pode estar relacionado à insuficiência do modelo biomédico de assistência à saúde. Este fato, aliado à frequência esporádica com que a mãe procura o serviço de saúde para atendimento da criança, chama a atenção para a necessidade de acompanhamento mais frequente pelos serviços básicos, por se tratar de crianças consideradas de risco ao nascer. Uma possibilidade por excelência consiste no acompanhamento pela Estratégia de Saúde da Família, criada em 1994, para prestar uma assistência integral, contínua, com resolubilidade e boa qualidade, tanto na unidade como no domicílio ${ }^{(15)}$, em especial, quando se trata de recém-nascidos de risco.

O maior tempo de aleitamento materno exclusivo como fator protetor para reduzir a morbidade na infância é corroborado pela literatura, pois evidências consistentes sugerem que a prática do aleitamento materno, sobretudo exclusivo, protege o recém-nascido contra doenças como diarreia, infecções gastrointestinais e outros sintomas de morbidade infantil, fornecendo uma série de outros benefícios para a saúde da criança, tanto a curto como em longo prazo ${ }^{(16)}$.

A ocorrência de óbitos no período neonatal precoce e pós-neonatal coincide com a tendência da mortalidade infantil brasileira. Estudo em Fortaleza - Ceará verificou que, dos óbitos neonatais de 2009, 79,5\% ocorreram no período neonatal precoce ${ }^{(17)}$. Em Cuiabá, em 2010, ocorreram 77 óbitos neonatais, dos quais 
$72,8 \%$ ocorreram no período neonatal precoce ${ }^{(18)}$.

Autores apontam a mortalidade neonatal precoce associada principalmente a um déficit na assistência prestada à gestante durante o pré-natal e parto, bem como à falta de atenção adequada aos recém-nascidos no momento do nascimento em unidades de terapia intensiva neonatal ${ }^{(17)}$. A mortalidade infantil, por constituir-se um grave problema de saúde pública, demanda investimentos na melhoria geral das condições de vida da população, incluindo a reestruturação da assistência prestada à gestante e recém-nascido, com articulação entre os serviços de atenção básica e os de assistência ao parto; garantindo o acesso igualitário à qualidade do pré-natal e ao processo de parto, nascimento e hospitalização do recém-nascido ${ }^{(19)}$.

Apesar do não ter sido encontrada associação entre número de consultas de pré-natal e óbitos, autores destacam que não fazer o pré-natal aumenta o risco para a ocorrência de perdas fetais, óbitos infantis e baixo peso ao nascer ${ }^{(20-22)}$. Assim, ressalta-se a importância da qualidade da assistência para identificação precoce das gestantes de risco ${ }^{(23)}$.

Embora a presente pesquisa não tenha evidenciado associação entre óbito nos seis primeiros meses de vida e escolaridade materna, autores apontam a relação desta variável com a sobrevida da criança, uma vez que tal associação pode estar relacionada ao baixo padrão socioeconômico das mães e, com isso, a possibilidade do baixo peso do recém-nascido, devido às mães iniciarem tardiamente o pré-natal e apresentarem menor ganho de peso na gestação ${ }^{(20)}$. Neste sentido, compreende-se que investimentos sociais, como na estrutura educacional do país, podem contribuir diretamente para a melhoria do cuidado à saúde.

Apesar da não associação entre óbito do recém-nascido com a pouca idade materna, autores apontam que filhos de adolescentes apresentam maior risco de morte no primeiro ano de vida ${ }^{(21)}$. Frente a isto, faz-se necessário que os profissionais de saúde criem vínculo efetivo com as mães adolescentes, incluído as famílias dessas jovens na assistência, favorecendo o diálogo e contribuindo para fortalecer o apoio familiar. Desta forma, apesar da pouca idade, a mãe poderá sentir-se com mais suporte e amparo no cuidado a si e ao recém-nascido.

O baixo peso ao nascer associado ao óbito também foi encontrado em Fortaleza - Ceará, entre recém-nascidos que vieram a óbito, $6,4 \%$ pesavam $<2.500 \mathrm{~g}^{(17)}$. O baixo peso ao nascer, decorrente seja de um crescimento intrauterino inadequado, seja de uma idade gestacional menor ou da combinação de ambos, constitui um deter- minante da mortalidade neonatal, perinatal e infantil, muitas vezes atrelado às condições socioeconômicas ${ }^{(0,22)}$.

O estudo não encontrou associação entre óbito até seis meses de idade e sexo do recém-nascido. No entanto, a literatura argumenta que recém-nascidos do sexo masculino têm duas vezes mais risco de vir a óbito do que o sexo feminino. Isto seria decorrente do amadurecimento mais precoce do pulmão fetal no sexo feminino, com a consequente diminuição de problemas respiratórios, uma das principais causas de óbito neonatal ${ }^{(22)}$.

\section{CONCLUSÕES}

O estudo analisou o perfil de morbimortalidade, nos seis primeiros meses de vida, entre recém-nascidos de risco, em Cuiabá - Mato Grosso, buscando possíveis associações entre a ocorrência de doenças e óbitos com variáveis maternas e socioeconômicas. Chama a atenção que as doenças que mais acometeram as crianças podem ser tratadas em atenção básica, onde podem receber diagnóstico precoce e tratamento adequado, evitando a evolução e o agravamento do quadro.

Prevenir as principais doenças nos primeiros anos de vida constitui-se um desafio, especialmente em grupos populacionais com limitadas condições socioeconômicas. Neste sentido, os resultados possibilitam refletir sobre a necessidade de investimentos na reestruturação da assistência prestada à gestante e recém-nascido, com articulação entre os diferentes níveis de atenção à saúde, garantindo o acesso e a qualidade do pré-natal, parto, nascimento e hospitalização do recém-nascido. Junto a estas medidas, mais e melhores investimentos na melhoria geral de vida da população se mostram essenciais, a fim de reduzir, efetivamente, os altos índices de morbimortalidade infantil em nosso país.

\section{REFERÊNCIAS}

1. Gorgot LRMR, Santos I, Valle N, Matisajevich A, Barros ALD, Albernaz E. Óbitos evitáveis até 48 meses de idade entre as crianças da coorte de nascimentos de Pelotas de 2004. Rev. Saúde Públ. 201;45(2): 334-42.

2. Ministério da Saúde (BR). Manual de vigilância do óbito infantil e fetal e do comitê de prevenção do óbito infantil e fetal. Brasília, [Internet] 2009 [acesso em 25 jul 2012]. Disponível: http://portal.saude.gov. br/portal/arquivos/pdf/Manual_Infantil_Fetal.pdf

3. Ministério da Saúde (BR). Sistema Nacional de Vigilância em Saúde. Relatório de situação de Mato

Cogitare Enferm. 2014 Jan/Mar; 19(1):109-15 
Grosso. Brasília, [Internet] 2009 [acesso em 25 jul 2012]. Disponível: http://portal.saude.gov.br/portal/ arquivos/pdf/005_mt_relatorio_de_situacao.pdf

4. Ministério da Saúde (BR). Agenda de compromissos para a saúde integral da criança e redução da mortalidade infantil. Brasília, [Internet] 2004 [acesso em 25 jul 2012]. Disponível: http://bvsms.saude. gov.br/bvs/publicacoes/agenda_compro_crianca.pdf

5. Ministério da Saúde (BR). Saúde Brasil 2008. 20 anos de sistema único de saúde (SUS) no Brasil. Brasília, [Internet] 2009 [acesso em 25 jul 2012]. Disponível: http://portal.saude.gov.br/portal/ arquivos/pdf/saude_brasil_2008_web_20_11.pdf

6. Ribeiro AM, Guimarães MJ, Lima MC, Sarinho SW, Coutinho SB. Fatores de risco para mortalidade neonatal em crianças com baixo peso ao nascer. Rev. Saúde Públ. 2009;43(2): 246-55.

7. Chalfun G, Mello RR, Dutra MVP, Andreozzi VL, Silva KS. Fatores associados à morbidade respiratória entre 12 e 36 meses de vida de crianças nascidas de muito baixo peso oriundas de uma UTI neonatal pública. Cad. Saúde Pública. 2009;25(6):1399-408.

8. Almeida MFB, Guinsburg R, Martinez FE, Procianoy RS, Leone CR, Marba STM, et al. Fatores perinatais associados ao óbito precoce em prematuros nascidos nos Centros da Rede Brasileira de Pesquisas Neonatais. J. Pediatr. 2008;84(4):300-7.

9. Moreira FM, Reis TLB, Cajado LCS. Fatores de risco a morbimortalidade infantil: desafios da Estratégia Saúde da Família. An Congr Bras Med Fam Comunidade [Internet] 2013;12:152 [acesso em 26 jun 2013]. Disponível: http://www.cmfc. org.br/index.php/brasileiro/article/view/685

10. Rosa AM, Ignotti E, Hacon SS, Castro HA. Análise das internações por doenças respiratórias em Tangará da Serra - Amazônia Brasileira. J Bras Pneumol. 2008;34(8): 575-82.

11. DATASUS. Ministério da Saúde/Secretaria de Atenção à Saúde (SAS): Sistema de informações hospitalares do SUS (SIH/SUS). DATASUS; 2012. [acesso em 25 jul 2012]. Disponível em: http://www2.datasus.gov.br/DATASUS/index.php

12. Paz MGA, Almeida MF, Gunther WMR. Prevalência de diarreia em crianças e condições de saneamento e moradia em áreas periurbanas de Guarulhos, SP. Rev. bras. epidemiol. 2012;15(1):188-97.
13. Ministério da Saúde (BR). Atenção integrada às doenças prevalentes na infância. Brasília, 2002 [Internet] [acesso em 25 jul 2012]. Disponível: http://bvsms. saude.gov.br/bvs/publicacoes/AIDPI_modulo_1.pdf

14. Bergamaschi SFF, Praça NS. Vivência da puérpera adolescente no cuidado ao recém-nascido, no domicílio. Rev Esc Enferm USP. 2008;42(3): 454-60.

15. Ministério da Saúde(BR). SaúdedaFamília: uma estratégia para a reorientação do modelo assistencial. Brasília, 1997 [Internet] [acesso em 25 jul 2012]. Disponível: http://bvsms.saude.gov.br/bvs/publicacoes/cd09_16.pdf

16. Ministério da Saúde (BR). Secretaria de Atenção à Saúde. Departamento de Atenção Básica. Saúde da criança: nutrição infantil: aleitamento materno e alimentação complementar. Série A. Normas e Manuais Técnicos. Cadernos de Atenção Básica, n. 23, 112 p. Brasília, 2009 [Internet] [acesso em 25 jul 2012]. Disponível: http://portalsaude.saude.gov.br/portalsaude/index.html

17. Nascimento RM, Leite AJM, Almeida NMGS, Almeida PC, Silva CF. Determinantes da mortalidade neonatal: estudo caso-controle em Fortaleza, Ceará, Brasil. Cad. Saúde Pública 2012;28(3):559-72.

18. Moreira MDS, Gaíva MAM, Bittencourt RM. Mortalidade neonatal: características assistenciais e biológicas dos recém-nascidos e de suas mães. Cogitare enferm. 2012;17(1):113-8.

19. Gaíva MAM. Atenção ao neonato na estratégia saúde da família: avanços e desafios para a atenção integral. Cogitare enferm. 2012;17(4):730-7.

20. Ramos HAC, Cuman RKN. Fatores de risco para prematuridade: pesquisa documental. Esc. Anna Nery. 2009;3(2):297-304.

21. Oliveira EFV, Gama SGN, Silva CMFP. Gravidez na adolescência e outros fatores de risco para mortalidade fetal e infantil no município do Rio de Janeiro, Brasil. Cad. Saúde Pública. 2010;26(3):567-78.

22. Carniel EF, Zanolli ML, Antonio MARGM, Morcillo AM. Determinantes do baixo peso ao nascer a partir das declarações de nascidos vivos. Rev. bras. epidemiol. 2008;11(1): 169-79.

23. Jobim R, Aerts D. Mortalidade infantil evitável e fatores associados em Porto Alegre, Rio Grande do Sul, Brasil, 2000-2003. Cad. Saúde Pública. 2008; 24(1):179-87. 